\title{
I microsismi come onde di Lamb-Rayleigh
}

\author{
(The micruseisms as Lamb-Rayleigh waves)
}

\author{
P. CHOOI $\left(^{*}\right)$
}

Ricevuto il 2 Gennatio 1973

\begin{abstract}
Russunto. - L'origine dei mierosismi viene spiegata, estendendo al loro insorgete la teoria di Lamb sulla propagazione di onde alla superficie di un mezzo soliclo elatico, provocate da una forza verticale, applierata alla superficie del mezzo. laz forzil, nel easo specifien, è eostituita da un fronte d'onla di una zona di mare, soggetta a "feteh". Le applieazioni ad aleune tempeste microsismiche, portano a valori per gli spostamenti, conformi a quelli previsti ialla teoria.

si jrova inoltre che i microsisui spotadici, aceiclentali, di carattere eschusivamente locale, ele insorgous contemporancamente ai microsigmi fondamentali, destinati alle srandi distanze, souo essenzialunente costituiti da onde longiludinali, ehe rientrann enue "frange" telle onde di Rayleigl, previste dalla tentia di Laumb.
\end{abstract}

Summary. - We explain the microseisms origin applying to their beginning the waves propagation Lamb's theory on the suffice of an elastic solid medium; waves ate cansed by a vertical force applied on the medium surface. In this case, the force is formed by a sea-zone wave front, sulject to "feteh". Applications to some microseisus storms bring forth values for displacements in conformity with theory.

We prove, besiles, that sporalie casual, exelusively local microsejems arising simultaneously with fundamental microseisms, destined to long clistances, are essentially formed by longitudinal waves, that come into Rayleigh waves "fringes", provided in the theory.

* Intituto Nazionale il Geofisica - lroma. 
1. - Xumerose sono le ipotesi a le teorie sullorigine dei microsismi. Fra esse, le più clilinse - per quanto eonerrue i mierosismi che originano in mare aperto o ai suoi limiti costieri - aftribuiscono le perturbazioni elastiche del terreno o all'urto tei marosi contro le eoste rocciose o all'azione tlei sistemi onclosi tlel mare sul fondo. Io non erelo che la prima sunsa sia molto efficiente al fine della creazione ali perturbazioni elastiche, con le aratteristiche di lunglezza d'onsla e sli propagazione, quali commemente si osservano in microsismi di origine marina (5); ritengo molto più rispondenti al vero le teorie che si riferiseono alla seconrla ipotesi (3.6).

Ciò premesso, vediamo quali possono essere le condizioni del mare, atte a slar origine sul fonclo a sistemi di onde rli tipo Rayleiglı.

2. - Richiami di Idrodinamica. Con riferimento ad un sistema di assi triortogonali $x, y, z$, se indichiamo con $X, Y, Z$ le componenti unitarie slelle forze di massa, secondo gli stessi assi, con on la rlensita slel mezzo (l'aequa, nel nostro easo) e con $P$ la pressiono unitaria, le equazioni generali del movimento di un sistema continno sono

$$
\frac{d^{2} x}{d t^{2}}=X-\frac{1}{\varrho_{1}} \frac{\partial P}{\partial x},
$$

e altre due anlaloghe per le direzioni $y, z$.

Nel movimento rli una massa fluida, si tratta quindi di lleterminare la posizione $x, y, z$ occupata in ogni istante rla una generica particella, supjonenrlo di conosecere la posizione e la velocita iniziale $\left(x=\frac{d x}{d i}, v=\frac{d y}{d i}, w=\frac{i z}{d t}\right)$ delle singole particelle e le forze che le sollecitiano.

poiche $\frac{d^{2} x}{d t^{2}}=\frac{d u}{d t}$, derivaurlo totalmente, le $\lceil 1\rceil$ slivengono

$$
\frac{\partial u}{\partial t}+u \frac{\partial u}{\partial x}+v \frac{\partial u}{\partial y}+w \frac{\partial u}{\partial z}=X-\frac{1}{u} \frac{\partial P}{\partial x}
$$

e rlue analoghe.

Se inclichiamo con $r$ il volume elementare sli una molecola fluidiu, per il principio dell'invariabilitì della massa $Q_{1} V^{r}$ durante il movimento, lequazione di continuità può seriversi

$$
\frac{d Q_{1}}{d t}+\varrho_{1} \frac{1}{V} \frac{d V}{d t}=0
$$


dove $d V$ rappresenta la variazione di volume che intervienc nella particella nel passaggio rlal tempo $t$ tempo tem $t d t$, intervallo infinitesimo al quale corrisponde uno spostamento infinitesimo tei singoli punti della massa, di componenti udt, vdt, wdt. Pertanto, la llilatazione abica corrispondente a detio spostamento surà

$$
\frac{1}{V} \frac{d V}{d t}=\frac{\partial u}{\partial x}+\frac{\partial v}{\partial y}+\frac{\partial u}{\partial z},
$$

c l'equazione di continuità prenilerà la forma

$$
\frac{d \rho_{1}}{d t}+\theta_{1}\left(\frac{\partial u}{\partial x}+\frac{\partial t}{\partial y}+\frac{\partial w}{\partial z}\right)=0
$$

che, per fluida incomprimibili si riduce a

$$
\frac{\partial u}{\partial x}+\frac{\partial v}{\partial y}-\frac{\partial w}{\partial z}=0
$$

Nel cho di moto staziontrio (o permanente), $u, v, *, o_{1}, P$ (consirlerate come funzioni di $x, y, z, t)$ sono costanti rispetto al tempo. Quancio $u, v, w$ derivano da un potenziale ri velocità $p(x, y, z, t)$, i

$$
u=\frac{\partial \varphi}{\partial x}, v-\frac{\partial \varphi}{\partial \eta}, w=\frac{\partial \varphi}{\partial z} ;
$$

per cui, per la [3], la $\varphi$ tovnà sorldisfare alla contizione

$$
\Delta \circ \varphi=0
$$

essemulo 1 , il laplaciano di $p$.

Dei moti rotati di potenziale di velocitì, a noi interessano naturalmente quelli caratterizzati da componenti nulle della velocitia angolare delle singole particelle $\frac{1}{-}\left({ }^{\partial *}-\stackrel{\partial v}{--}\right), \ldots$ : cioes, i mote irotazionali.

Supponendo le forze conservative, intionndo con $U$ il potenziale unitario cper eni $X=\frac{\partial U}{\partial x}, Y=\frac{\partial U}{\partial y}, Z=\frac{\partial U}{}$, , le [1'] - nell'esistenza uli un potenziale di velocita e tenendo conto delle [t]-, con trasformazioni evidenti, si riassumono nella relazione

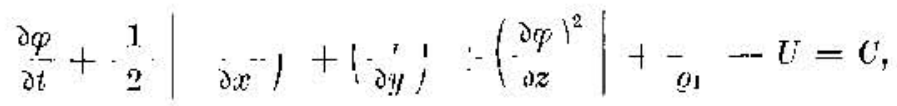


dove $($ rappresenta la costante d'integrazione (rispetto ad $x, y, z$ ), che, in generale, puo dipendere dal tempo.

Consirteriłmo ora il caso, che a noi più interessa, di un moto oseillatorio nel piano vertioale $x z$, con l'asse $z$ rivolto verso l'alto. Se il moto è stazionario, la $[6]$ diviene

$$
\frac{1}{2}\left\{\left(\frac{\partial \varphi}{\partial x}\right)^{2}+\left(\frac{\partial \varphi}{\partial y}\right)^{2}\right\}+\frac{P}{\rho_{1}}-I=C,
$$

dove $C$ e una costante eflettiva. Jaa [7] e la ben nota equazione di Bernoulli.

Nel caso in esame, il potenziale della forza ̀̀

$$
\zeta=-g z,
$$

dove $q$ è l'aceelerazione di gravitì. Dalla [7], prescinclendo da $C$, otteniamo per la pressione l'espressione:

$$
P=\varrho_{1}\left\{-g z-\frac{1}{2}\left[\left(\frac{\partial \varphi}{\partial x}\right)^{2}+\left(\begin{array}{c}
\partial \varphi \\
-\partial z
\end{array}\right)^{2}\right]\right\},
$$

dove $\varphi$, in forza della [5], sodrlisfa alla relazione

$$
\frac{\partial^{2} \varphi}{\partial x^{2}}+\frac{\partial^{2} \varphi}{\partial z^{2}}=0
$$

Siano $h_{1}$ la profonditi media della massa liquida o a lampiezza del moto oudulatorio in superficie.

Nel caso di moto stazionario, sovrapposto ad ma corrente uniforme di velocitł c, un potenziale di velocità che sorddisfa la [9] ì

$$
\varphi=c\left(-x+a \frac{\cosh k_{1}\left(z+h_{1}\right)}{\sinh k_{1} h_{1}} \sin h_{1} x\right),
$$

dove è $k_{1}=\frac{2 \pi}{\lambda_{1}}$, essendo $\lambda_{1}$ la lunghezra d'onda o $c$ è la velocità di propagazious lungo l'asse $x$.

Ia pressione sul fondo è data dalla [8]. I casi chlo a noi interessano nel seguito sono caratterizzati da grandi valori di $\lambda_{1}$ (e da piesole profoulliti), per eui nell'espressione di $\left(\frac{\partial \varphi}{\partial x}\right)^{2}-\left(\frac{\partial \varphi}{\partial z}\right)^{2}$ possono essere trascurati $\mathrm{i}$ termini in $k_{1}{ }^{2}$. Si ha allora dalla [8], per $z=-h_{1}$,

$$
P=\varrho_{1}\left\{g h_{1}-\frac{r^{2}}{2}\left\{1-\frac{2 k_{1} a}{\sinh h_{1} h_{1}} \cos h_{1} x\right\}\right),
$$

essendo $\cosh 0=1$. 
Ia determinazione di $c$ presuppone moto non permanente $\left(\frac{\partial p}{\partial i}, 0\right)$ : nel nostro caso, eome si è tletto, un moto periodico pispetto at $x$, sovrapposto ad una corrente uniforme di velociti c. Alla superticie libera rlell'acqua $(z=(1)$, vale allora la contizione di Poisson

$$
\frac{\partial^{2} q}{\partial t^{2}}+y \frac{\partial \varphi}{\partial z}=0
$$

la quale consente ti ottenere

$$
c=\sqrt{\frac{g \hat{h}_{11}}{2 \pi} \cdot \text { tangh } k_{1} h_{1}} .
$$

Avremo quiudi

$$
c^{2}=g h_{1} \frac{\operatorname{tangh} k_{1} h_{1}}{h_{1} h_{1}}
$$

Sarà perciò

$$
P=\varrho_{1} g\left|h_{1}+\frac{a}{\cosh h_{1} h_{1}} \cos k_{1} x-\frac{h_{1}}{2} \frac{\text { tangh } h_{1} h_{1}}{h_{1} h_{1}}\right| .
$$

Ma, per $h_{1}$ piccolo nei confronti di $\lambda_{1}$, tangh $h_{1} h_{1}$ tente $a k_{1} h_{3}$ e quiucli

$$
c^{2}=g h_{1}
$$

A meno rli una costante alslitiva, $i$ pertanto

$$
P=\varrho_{1} q\left[h_{1}+\frac{a}{\cosh h_{1} h_{1}} \cos k_{1} x\right] .
$$

L'iucremento $A P$ di pressione sul fonto, assoriato al moto onsloso rli ampiezza $a$, risulta percio uguale a quello ri uua colonna t'acqua oscillante di altezza $\frac{a}{\cosh h_{1} h_{1}}$.

A questat conclusione era già arrivato, per altra via, (1. Amtreotti ${ }^{1}$ ).

Facciamo delle applimazioni valevoli per l'alto Alriatico (golfo di Venezia). La zona dove - quaurlo le condizioni si presentano - insorgono microsismi, ì quella a cavaliere del golfo tli Trieste (3).

Per $h_{1}=15 \mathrm{~m}$ ¿ $c=\sqrt{g h_{\mathrm{l}}}=12,13 \mathrm{~m} / \mathrm{sec}$. Stabilitosi un regime stazionario, per "fetch " pienamente svilupuato, il periorlo dei micro- 
sismi is tl 3 sec a. Consegue $\lambda_{1}=36,4 \mathrm{~m}$. Avremo quindi $\frac{1}{\cosh k_{1} h_{1}}$ $=0,15$. Fatto $a=2 \mathrm{~m}$, consegue

$$
A P=Q_{1} f \frac{a}{\cosh k_{1} h_{1}}=300 \mathrm{~kg} / \mathrm{m}^{2}
$$

cioè una pressione dell'ordine rli $3 \cdot 10^{5}$ tonnellate per kmq.

Per $a=3 \mathrm{~m}$, risulta

$$
A P=450 \mathrm{~kg} / \mathrm{m}^{2},
$$

e quindi una pressione sul fondo dell'orrine fli $4,5 \cdot 10^{5}$ tonnellate per kma.

3. - Consideriamo un solislo elastico, semi-infinito, limitato dal piano $z=0$ e giacente dal lato positivo di questo piano (nel nostro (uso, il fonto alel mare). Fra le canse capaci di viestare nel mezzo elastico ontle di Rayleigh, vi is quella costituita da una forza vorticale $\theta \cdot e^{i p t}(p)$ pulsazione della forza agente) applicatta alla superfieie del mezzo, parallelamente a $z$, e concentrata nei punti della linea $x=0$, $z=0$. Il caso ì stato risolto dta Horace Lamb. Indicando con $T$, $v_{3}$ e $\lambda$ rispettivamente il periodo, la velocità di fase e la lunghezza r'onda dell'onda fli Rayleigh, se $u_{o}$, $v_{0}$ esprimono lo spostamento seconcio $x$ (direzione di propagazione) e seconrlo $z$, Lamb (10) otfiene:

$$
\begin{aligned}
& u_{0}=-\frac{\theta}{\mu} H e^{i n\left(i-\frac{i}{z_{3}}\right)}+\frac{Q}{\mu} \sqrt{\frac{2}{\pi}} \sqrt{\left(1-\frac{h^{2}}{k^{2}}\right)} . \\
& \cdot \frac{e^{i\left(p t-k x-\frac{1}{l} \pi\right)}}{(h x)^{3 / 2}}-\frac{Q}{\mu} \sqrt{\frac{2}{\pi}} \frac{h h^{3} k^{2}}{\left(h^{2}-2 h^{2}\right)^{3}} \cdot \frac{i e^{i\left(p t-h x-\frac{1}{4} \pi\right)}}{(h x)^{3 / 2}}+\ldots \\
& \tau_{0}=-i \frac{Q}{\mu} K e^{i p\left(l-\frac{x}{v_{3}}\right)}+2 \frac{\vartheta}{\mu} \sqrt{\cdot \frac{2}{\pi}}\left(1-\frac{h^{2}}{k^{2}}\right) \\
& \frac{i e^{i\left(p t-k x-\frac{1}{1} \pi\right\}}}{(k x)^{3 / 2}} \div \frac{Q}{2 \mu} j^{i \frac{2}{x}} \frac{h^{2} k^{2}}{\left(k^{2}-2 h^{2}\right)^{2}} \cdot \frac{i e^{i\left(p t-h x-\frac{1}{4} \pi\right)}}{(h x)^{3} /}+\cdots
\end{aligned}
$$

flove:

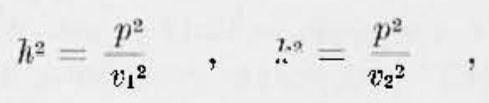


essendo $v_{1}, v_{2}$ le velocità di propagazione delle onde longitudinali e trasversali proprie del mezzo; e quindi

$$
v_{1}^{2}=\frac{\lambda+2 u}{\varrho} \quad, \quad v_{2}^{2}=\frac{\mu}{\varrho}
$$

in cui $\lambda, \mu$ sono le costanti di Lamé e $g$ la densità del mezzo. Com'è noto, per $\sigma=\frac{1}{4}$ ̀̀

$$
v_{3}=0,9194 \cdot v_{2}
$$

$H$ e $K$ sono costanti dipendenti da $h^{2}, k^{2}$ e $A=T v_{3}(T$, periodo dell'onda elastica); dipendono quindi dal coefficiente $\sigma$ di Poisson. I amb trova che il loro valore cresce da $\sigma=\frac{1}{2}$ (incompressibilitì) a $\sigma=0$. Per $\sigma=1 / 4$, ̀

$$
H=0,12500, K=0,18349 \text {. }
$$

E evidente che, nelle [12], tutti i termini dopo il primo diminuiscono indefinitamente al crescere di $x$. Pertanto, a distanze sufficientemente grandi dall'origine della perturbazione, per $x$ positivo le [12] si riducono a

$$
u_{n}--\frac{Q}{\mu} H e^{i p\left(t-\frac{x}{v_{3}}\right)} \quad, \quad v_{0}=-i \frac{Q}{\mu} K e^{i p\left(t-\frac{x}{v_{3}}\right)} .
$$

Similmente, per $x$ negativo, si troverebbe

$$
u_{0}=\frac{Q}{\mu} H e^{i\left(1+\frac{x}{v_{3}}\right)} \quad . \quad v_{0}=-i \frac{Q}{\mu} K e^{i p\left(+\frac{x}{r_{3}}\right)} .
$$

Queste formule rappresentano un sistema di oncle libere cli Payleigh, ad eccezione per la discontinuitì all'origine, dove la forza estranea è applicata. Le vibrazioni sono ellittiche, con assi orizzontale e verticale nel rapporto dei numeri $H$ e $K$ (che è il rapporto di Rayleigh).

Con riferimento alle [14], le parti reali di $u_{o}, v_{o}$ si scrivono

$$
u_{0}=-\frac{Q}{\mu} H \cos p\left(t-\frac{x}{v_{3}}\right) \quad ; \quad v_{0}=\frac{Q}{\mu} K \sin p\left(t-\frac{x}{v_{\mathrm{a}}}\right) \text {. }
$$

4. - Consirleriamo il caso di una zona di mare poco profondo. Il passaggio di un'area ciclonica, abbia lasciato la superficie del mare in stato di "fetch", con sistemi di onde stazionarie di ampiezza $a$ 
pressoclé eostante (5). Sia $x$ la direzione di propagazione fli un sistmna ondoso: noi possiamo identificare ogni clemento di questo sistema, come la linea $x=0, z=0$, in ogni punto rella quale - paralielamente a $z$ - agisee la forza $O$. Abbiamo già visto al n. 2 che, in caso fi mare poco profondo, l'incremento di pressione sul fondo, associato al moto omiloso di ampiozza a, può essere espresso da.

$$
A P=\theta 1 y \frac{a}{\cosh k_{1} h_{1}} .
$$

Se $y_{1}, 7_{2}$ sono le coordinate tegli estremi di un fronte d'onda, sarà

$$
Q=A P \int_{y_{1}}^{y_{2}} d y .
$$

L'attendibiliti o meno di queste argomentazioni prò essere attestata solo dalle applicamioni.

$\Lambda$ questo scopo, riferiamori a tempeste mirrosismiche originanti in zone di mare ben delimitate, oggetto di precedenti indagini (3-6).

Esaminiamo dapprima il easo dei microsismi che nascono dal foncio rlell'alto Atriatico, con particolare riguardo al golfo di 'Trieste. Una tempesta curatieristica fu fuclla ale 15 Lnglio 1970 (1-6). Fissa fu registrata in un ampio raggio, ta Trieste a Vienna.

Innanzi tutto, dobbiamo veriere se, nelle [12], possono essere traseurati i termini flel secondo membro, snecessivi al primo.

Possiamo considerare Trieste - stazione sismica più prossima alla zona origine - alla tistanza approssimativa di $20 \mathrm{~km}$ ta detta zona.

Nell'intervallo di massimo svilupno delle onde significative, il periorlo dei microsismi, nella zona in questione, è di circa 3 sec. $\mathrm{Ab}$ biamo quindi

$$
h^{2}=\frac{4,41}{v_{\mathrm{r}^{2}}} \quad, \quad k^{2}=\frac{4,41}{v_{2}^{2}}
$$

Ricordiamo che il fonto dell'Adriatico consiste di selimenti ali Aluvionale, Pliocenico, Miocenico e Oligocenico-Focenico, nell'ordine, fino al sottostante Cretaceo, per nno spessore merlio, nella zona considerata, di un migliaio di metri eirca (3). Le velocità delie onde longitudinali e trasversali in mezzi tanto compositi, variano entro limiti estesi e, comunque, crescono dall'Aluvionale (attuale fondo flel mare) 
all'Eocenico. İ ta ritenere però che solo gli strati più superficiali vengano interessati da microsismi aventi un perioulo massimo di soli 3 sec (1); per cui, possiamo ragionevolmente ritenere dellordine di $2 \mathrm{~km} / \mathrm{sec}$ la velocita tlelle onde longritulinali e di poeo sujeriore ar $1 \mathrm{~km} / \mathrm{sec}$ quella delle trasversali.

Nell'ipotesi di Poisson, per convenienza di calcolo possiamo porre $v_{1}=1732 \mathrm{~m} / \mathrm{sec}, v_{2}=1000 \mathrm{~m} / \mathrm{sec}$.

Con questi dati di partenza, avremo

$$
h-1,21 \cdot 10^{-5} ; k=2,1 \cdot 10^{-5} ; \mu=2 \cdot 10^{10} \text { e.g.s. }
$$

Tertanto, per Trieste risulta

$$
h x=24,2 ; k x=42 .
$$

Prescintendo dal rapporto $\frac{\hat{\gamma}}{\mu}$, avremo quindi per il 20 e il $3^{\circ}$ termine di $n_{0}$ nella [12], rispettivamente:

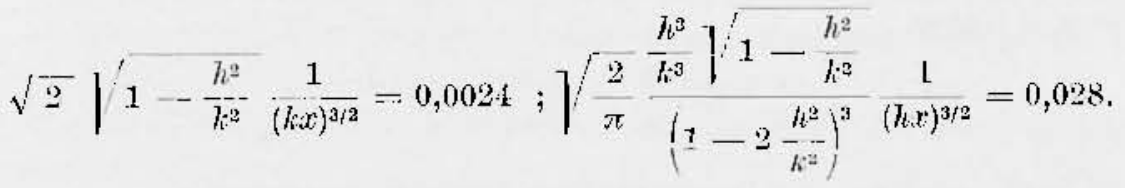

F, analogamente, per il $2^{\circ}$ e $3^{\circ}$ termine ti $v_{v}$ :

$$
\begin{aligned}
& 2 \frac{2}{\pi}\left(1-\frac{h^{2}}{h^{2}}\right) \frac{1}{(k x)^{3 / 2}}=0,0039 ; \\
& \frac{1}{2} / \frac{2}{\pi} \cdot \frac{\frac{h^{2}}{k^{2}}}{h^{2} / 2} \frac{1}{(h r) 3 / 2}=0,01005 .
\end{aligned}
$$

Tenuto conto tlei valori di $H, K$ dati rlalla [13], i contributi dei Lermini suceessivi al primo, nelle espressioni di $u_{o}$ e $v_{0}$ possono quindi essere trascurati.

Cii, naturalmente, sarà tanto più vero quanto più cresce la distanza ılella stazione sismica. Per Parlova, p. es., a circa $130 \mathrm{~km}$, si ottengono per i coeflicienti di $-\mu_{\mu}$ nel $2^{\circ}$ e $3^{\circ}$ termine di $u_{\infty}$, i valori

$$
0,00014 \text { e } 0,001716
$$


rispettivamente; mentre, gli analoghi valori in $r_{0}$ divengono:

$$
0,00024 \text { e } 0,00061
$$

rispettivamente; trascurabili quincli nei confronti di $H$ e $K$.

Calcoliamo ora il valore di $Q$, nelle ipotesi fatte.

Per la zona in considerazione, possiamo porre $h_{1}=15 \mathrm{~m}$, da cui segne $\lambda_{1}=36,4 \mathrm{~m} ; \frac{1}{\operatorname{coshl} k_{1} h_{1}}=0,15$. Essendo $o_{1}=1$, per $a-1,5 \mathrm{~m}$, si ottiene $\Delta P=22072,5$ dine $/ \mathrm{cm}^{2}$.

Per la scelta del valore dia assegnare alla difterenza $y_{2}-y_{1}$, ciò̀ alla lunghezza delin linea, in ogni punto della quale agisce la forza $Q$ sul fondo, in 1 piano vertieale, possiamo rilevare t]le ogui sistema d'onde stazionario procerte su fronti d'onda di diversa lunghezza: una stima media, accettabile, di tale lunghezza porta a ritenere $y_{2}-y_{1}-100 \mathrm{~m}$. Si ha quindi $Q=22072,5 \cdot 10^{4}$ dine $/ \mathrm{cm}$.

Il coefficiente di $\cos p\left(t-\frac{x}{v_{3}}\right)$, nell'espressione di $u_{0}$, assume alloria il valore

$$
H \frac{Q}{\mu}=1+\text { micron; }
$$

in modo analogo, per la componente verticale $v_{0}$ avremo

$$
K \frac{Q}{\mu}=20 \text { micron. }
$$

A parità di altre condlizioni, per $a=2 \mathrm{~m}$, si trova

$$
H{ }_{\mu}^{Q}=18 \text { mirron; } \quad K \frac{?}{\mu}=27 \text { mieron. }
$$

Un'alta scnsibilissima zona di mare, sorgente di microsismi, ¿̀ quelia che si estende fra La Spezia, le isole di Pianosin, Montecristo e del Giglio, comprendendo la dorsale dell'Isola d'Elba. Si tratta di un'area marina di almeno $6.000 \mathrm{kmq}$, di acque relativamente basse. Ja profondità media può essere valutata sui $90 \mathrm{~m}$.

Attesa la vastità della zona, il periorlo rlelle onde signilicative può ivi variare da 4 a 5 sec, per ragginngere, nel pieno sviluppo, i 6 sec (figg. 2, 3). Consideriamo il valore mexlio di 5 sec. Arl una lunghezza d'onda $\lambda_{1}=150 \mathrm{~m}$ corrisponde, sulla base della media profonditi considerata, 
$\frac{1}{\cosh k_{1} h_{1}}=0,05$. Pertanto, fatito $a=4 \mathrm{~m}, y_{2}-y_{1}=100 \mathrm{~m} \mathrm{e}$ $\mu=2 \cdot 10^{10}$, consegue

$$
H \frac{Q}{\mu}=12,3 \text { micron; } \quad K \frac{Q}{\mu}=18 \text { mieron, }
$$

il secondo valore esprimendo la componente verticale.

Altre zone di mare attivissime sono le distese ocenniche, prossime alle coste europee del Ford Allantico. La zona prospicente la Senndinavia e il Hare del Nord dà origine a microsismi di notevole energia, con periodi dell'ordine di 5 - 6 sec.

Ma, almeno per quanto riguarda le stazioni sismiche sparse sul continente etropeo, dove i microsismi insorgono piu violenti e piti frequenti, ̀̀ senza dubbio nella fascia atlantica che si svolge all occidente delle Isole Britanniche, dell'Irlanda e della Francia fino a Biarritz, presso i confini con la Spagna (fig. 4). E un'estesissima area di mare, larga dai 50 ai $500 \mathrm{~km}$, carntterizzata da piccole profonditiz e limitata al oceidente dlallisolata di $200 \mathrm{~m}$. Il periodo medio dei microsismi, legati ai sistemi di onde significative, può variare fra $\mathrm{i}$ $\tau$ e i 9 sec, il più frequente essenclo di 8 sec ca.

Valutiamo l'ordine di grandlezza delle ampiezze massime di tali microsismi.

Per una profondità media di $100 \mathrm{~m}$, al periodo di 8 sec corrisponde una lunghezza d'onda di $250 \mathrm{~m}$ circa. Si la quindi $\frac{1}{\cos l_{1} k_{1} h_{1}}=0,1615$. Per un'ampiezza d'onda $a=3 \mathrm{~m}$ e per un fronte d'onda dell'orline di $100 \mathrm{~m}$, si ottiene

$$
Q=4753 \cdot 10^{5} \text { c.g.s.s. }
$$

Per $\mu=2 \cdot 10^{10}$ (come per i due casi precerlenti), tenendo ronto dei valori di $H$ e di $K$, avremo per la componente orizzontale e verticale rispettivamente

$$
{ }^{Q} \cdot H=30 \text { micron, } \frac{Q}{\mu} K=44 \text { micron. }
$$

Probabilmente, per la zona in esame, costituita dallo zoceolo continentale, il valore di $\mu$ sopra riportato ì troppo piccolo, sia per un più limitato spessore dei sedimenti, sia per la maggior lunghezza d'onda dei microsismi, che li porta at interessare un più notevole spessore della crosta terrestre. Un valore attendibile per la velociti 
delle onde trasversali nugli strati esterni è quello di $t_{2}=2 \mathrm{~km} / \mathrm{sec}$, a eni corrispontle $\mu=\mathrm{s} \cdot 10^{10}$ e.g.s. Con questo valore per $\mu$, si ottiene

$$
\frac{\vartheta}{\mu} H=7,4 \text { micron, } \quad \frac{Q}{\mu} K=11 \text { micron. }
$$

Ricordiamo che, quando quei tratti di mare sono investiti dalle vaste e profomle are cicloniche provenienti dall'Atantico occidentale, si sviluppano onde amplissime, flell'ordine di $20 \mathrm{~m}$ tra massimo e minimo (r). In questi casi - che sono i più frequenti - è lecito porre (1. $.5 \mathrm{~m}$. Si ottiene allora

$$
\begin{array}{ccc}
\text { per } \mu=8 \cdot 10^{10} & \text { per } \mu=2 \cdot 10^{10} \\
\frac{Q}{\mu} H & 12,4 \text { micron } & 49,5 \text { micron } \\
\frac{Q}{\mu} K & 18,2 \quad \text { " } & 72, \overline{7} \text { " }
\end{array}
$$

it da ritenere che l'ampiezta dei microsismi abbia un valore intermedio ira quelli riportati.

5. - Sulla base delle osservazioni degli spostamenti provocati da tempeste microsismiche in diverse localiti, si pui senz'altro aflermare che i valori ottenuti al $n . \&$ sono attendibili, come del resto avremo morlo dli velere più avanti, nel confronto dei valori osservati per tempeste microsismiche, provenienti rlalle zone prese in esame.

Fa osservato che - giusta l'ipotesi prospettata - in realtà i valori calcolati nel numero precedente, sono valirli per difetto. Sul foudo del mare non agisce infatti soltanto un singolo fronte d'onda, cui compete lo spostamento dell'orcline sopra riportato, bensì un mumeroso sistema di oude, ognuma delle quali, contemporanemente, porta il proprio contributo sul foudo.

La fase iniziale di una tempesta microsismica è sempre costituita la oscillazioni di piecola ampiezza e di periodo sensibilmente minore di quello prefominante in wu sistema di onde significative, completamente sviluppato. Una voita stabilitosi nu sistema di onle stazionarie, sopra una linea determinata del fondo agisce ritmictamente nn'onda - con periodo ali $3,5,8, \ldots$ sec, a secomla della zona di mare interessata dall'area ciclonisa in trunsito - che ripetenslo, con il suo ritmo, la sua azione provoca uno stato di regime sul fondo. Temuto conto dell'elevata velocità dell'onda solicla propagantesi nel mezzo sotto- 
stante, e considerando cle, in fase con quella presa in esime, altre oscillazioni marine isocene agiseono a distanze pari a multipli di una lunghezza d'onda, l'onda solidir su tletta, in un intorno che potrì rariare fra qualche centinaio e qualche mixliaio di metri, subirà tliecine di eontributi, siechí l'oscillazione risultante sarà, per il principio flella sovrapposizione dei piecoli moximenti, la sommib delle ampiezze di ogni singola ondir.

Fsiste insomma - in certe direzioni e in certi spazi - una reciproca eccitazione delle singole onde solide; mentre, in altre direzioni e in altri settori, si verifieleranno fenomeni di interferenza. IDi qti laspetto di irregolari battimenti — irregolarita ampiamente motivata - sotto eui si presenta la registrazione dei microsismi.

Questo meceanismo origine, oltre a spiegare lordine tli grantezza degli spostamenti osservati, giustifica quinti anche la forma tlei treni ti microsismi, quale viene registrata; e cioè il susseguirsi di battimenti tlifformi e ti inegnal numero di elementi. Se poi si la la dovuta attenzione al fatto che, sotto l'azione del vento e - più ancora - delle microvariazioni della pressione atmosferica interagenti eon l'acqua, i fronti d'onla (e quindi le linee flazione della forza periotioa sul fon(lo) possono ruotare sulle primitive direzioni, si la in ció un'ulteriore piena giustificazione ti tute le mutazioni di forma e variazioni dampiezza osservate. Sjicga infine quanto sia vano ricereare - come ¿̀ stato più volte tentato - nell'anlamento e nel moto risultante dei microsismi quelle conferme sperinentali, che solo possono consentire fenomeni di origine fissa e al azione istantanea.

1)etto questo, esaminiamo i valori osservati in the tempeste microsismiche, precerlentemente esaminate $\left({ }^{5,0}\right)$.

Tenuesta microsismica tlel 15 Juglio 1970: zona-origine, il golfo di Trieste. Ampiezzc dei microsismi - aspresse in misron - con periotlo tli $2, \pi$ sec ca, osservate a:

\begin{tabular}{|c|c|c|c|c|c|c|c|c|c|}
\hline Trieste: & (allitit & tistanza & & 10.20 & ini & i & mieron & (rosili). & vert.) \\
\hline Somplago & $(n$ & $"$ & » & $8 \overline{7}$ & $n$ & 1,5 & " & $(1)$ & oriz.) \\
\hline Ial Mlaina & $(n$ & " & " & 110 & $"$ & ) 0,3 & $n$ & $(1)$ & verti.) \\
\hline P'ieve di Cadore & $(*$ & " & 1 & $12 \overline{7}$ & $*$ & $1 . \mathrm{L}$ & $"$ & * & $n \quad)$ \\
\hline Padoval & $(3$ & $"$ & 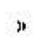 & 330 & $"$ & 15 & $"$ & $n$ & oriz.) \\
\hline Bologria & $(*$ & $"$ & $n$ & 200 & " & ) 6 & $"$ & $"$ & $n)$ \\
\hline Zagaliria & $(n$ & $"$ & $n$ & 200 & $n$ & 0,75 & $"$ & $n$ & vert.) \\
\hline Yienna & $1 n$ & $"$ & $n$ & 355 & $"$ & 0,2 & $n$ & $"$ & $n$ ) \\
\hline
\end{tabular}


Dove funzionava, la componente verticale ha clato i maggiori spostamenti. I valori si riferiseono ai massimi, osservati nelle varie stazioni, verso le 18 e 30 .

Dallesame di detti valori, scaturisee netta una fonlamentale constatnzione: la notevole lisparità degli spostamenti rilevati nelle diverse direzioni. Isa spieguzione si trova nella diversa natura geologica degli strnti interessati dai mierosismi. Come si è visto, questi insorgono sul fondo dell'alto Adriatico, verso il golfo di Trieste, dove i sedimenti presentano uno spessore complessivo di $700-800 \mathrm{~m}\left(^{3}\right)$. E da ritenere, fattesa la breve Iunghezza d'onda dei microsismi, che questi siano confinati nei 400-500 metri esterni. A mano a mano che si procele verso Trieste, le stratificazioni alluvionali si assottigliano, per annullarsi presso le rive del golfo. I microsismi registrati a Trieste hanno quindi subito una prima rifrazione, nel passaggio dagli strati alluvionali al mezzo cristłallino, su cui poggia la stazione sismica. Ciò sppiega perché a Trieste l'ampiezza tlei microsismi sia notevolmente più piccola di quella registrata a Padova, ad una distanza molto maggiore. Infatti, il fondo dell'Ailriatico ì continuato, senza alterazioni sensibili, nella Valle Parlana, sicché lił propagazione delle oncle elastiche si verifica senza attenuazione apprezzabile, se non quella legata alla distanza; il che spiegta le notevoli ampiezze osservate a Padova, nonelió il fatto che a Bolognł, a cirea $200 \mathrm{~km}$ talla zona origine, l'ampiezza dei microsismi sia ancora dell'ordine di 6 mieron, pari cioè a quella registrata a Trieste, ai margini della zona attiva. I microsismi registrati in tutte le altre stazioni, oltre all'attenuazione associata alla distanza, subiscono una perdita di energił più o meno sensibile, in corrispondenza del passaggio dagli strati alluvionali a quelli rocciosi flei sistemi di montagne. In particolare, la riduzione di energił si presenta notevole nell'attraversamento della profonda, insabbiata valle dell'alto Tagliamento, il che giustifica la piccol $₫$ ampiezz $₫$ dei microsismi registrati a Ia Maina (Samis), a quota $1.000 \mathrm{~m}$ s.l.m.

La tempesta in esame si esaurisce nel raggio di $370 \mathrm{~km}$ ca.

J'intensità ben maggiore sono le tempeste microsismiche, che originano ad occislente delle isole Britanniche e lungo lo zoceolo continentale atlantico.

Nel prospetto che segue, sono riportati gli spostamenti massimi in micron, provocati dai microsismi provenienti dł dletta zona, sul ritmo di 8 sec ea, e registrati verso le 15 del 20 Gennaio $1971\left(^{\circ}\right)$ nelle stazioni sotto indicate, alle distanze approssimative segnate di fianco al nome ilelle stazioni stesse: 


\begin{tabular}{|c|c|c|c|c|c|}
\hline Ojorto (Porto) & $800-900 \mathrm{~km}$ & comp. & vert. & 16 & micron \\
\hline Toledo & $1.000 "$ & $"$ & $"$ & 12 & $"$ \\
\hline Roma C.U. & $1.800 "$ & $"$ & $n$ & 1,1 & $n$ \\
\hline Uppsala & $2.000 \%$ & y & $"$ & 2,5 & $n$ \\
\hline
\end{tabular}

Ja quella zona provengono tempeste microsismiche, che sviluppano energie nettamente maggiori di quella qui esaminata, e che confermano l'attendibilità dei valori teorici, riportati al n. 4. Tempeste del genere (fig. 4) possono essere sensibili (sotto forma di microsismi) fino a $5.000 \mathrm{~km}$ e più.

6. - ફ̀ già stata tiscussa la controversia, relativa alla correlazione fra periodo dell'onda marina e periodo tel microsisma ad essa legato(5,6). Fino a vent'anni or sono, vi era chi attribuiva i microsismi eschusivamente (12) al un effetto di second'ordine, legato al sistema di ontle stazionarie agenti nell'area ciclonica, e ehi recisamente lo negava $\left({ }^{9}\right)$. Oggi è accettato l'mo e l'altro meceanismo (5): l'azione tlella doppia frequenza, conformemente alla teoria di Longuet-Higgins, è riservata al mare aperto, profondo; la frequenza primaria è limitata alle zone di mare poco proíondo (mari interni, o zoccoli eontinentali oceanici), dove le onde agenti "sentono il fondo", come nei casi qui esamillati.

E da sottolineare, ancora una volta, lo stretto legane fra microsismi ed interazioni fra atmosfera ed idrosfera nelle zone di mare soggette al transito dei cicloni: quando le depressioni si propagano con velocitì paragonabili a quelle delle onde libere del mare sottostante, insorgono fenomeni li risonanza, in cui le onde significative vengono eccitate da coperiodali variazioni microbariclı; ì questa una delle cause più efficienti per l'insorgere dei microsismi $(3,5, y)$.

7. - In un recente lavoro (1), è stato provato - con carattere di priorità - che microsismi insorgono ai margini immeliati di piccoli bacini, quando su questi transitano disturbi microbarici, anche estremamente labili, associati o no ad aree depressionarie.

In questi easi, naturamente, non ì consentito parlare di "fetch ", né di interazioni fra aria ell acqua comportanti fenomeni di risonanza. I microsismi cosi originati hanno infatti vita brevissima e si presentano sotto le gamme ritmiche piǹ disparate: iे tutto un susseguirsi di im- 
pulsi, generalmente con periodi inferiori al seconlo (ligg. 1,5 ), sebbene sovente preedano movimenti eon poriodi dell'ordine di $5 \mathrm{e}$ più seeoneli $\left({ }^{6}\right)$.

Pertanto, ì da ritenere che l'energia dhe anima questi mierosismi sia sostanzialnente quella inelota nellacpua dall'energia associata alle rapide variazioni di pressione, in transito sal lago: in altri termini, sono essi i microsismi sportadici, tempornei, non legati a sistemi di onde signifuetive, dei quali ho parlato in precedenti lavori (1.5. 5).

Possono rientare questi microsismi nella teoria riassunta al n. 3? Osiserviamo che, per $x=0$ (distanza nulla), le [12] presentano una discontimutà all'orisfine, flove ì applicata la for'za agente. Il primo termine delle $[12]$ ì già stato interpretato e conduce alle onde di Rayleigh. Per quanto rigruarda la perturbazione resirlua, fliremo con Jamib (l.c., pag. 21), che essa costituisce nua specie di frangia alle onde elastiche eilinuriche, che sono propagate nell'interno del solislo. Essa cousiste di due parti. In una di queste, la velocità di fase $p / k=v_{2}$, ¿ quella dielle ourle trasversali: alla superticie, le vibrazioni sono ellittiche, il rapporto rell'asse verticale a quello orizzontale dell'orbita essendo - $\mathrm{ger} \sigma=1 / 4$

$$
2 \longdiv { 1 - \frac { h ^ { 2 } } { k ^ { 2 } } } = 2 \longdiv { 1 - \frac { v _ { 2 } ^ { 2 } } { v _ { 1 } ^ { 2 } } } = 2 \longdiv { \frac { a } { 3 } } = 1,63330 \text {. }
$$

Ja parte rimanente, la la veloetit di fase $p h=n$, dolle onde longitulinali: le vilmazioni superficiali che essa rappresenta sono rettilinee, il rapporto rlellampiezza verticale all'orizzontale essendo sempre per $\lambda=\mu-$

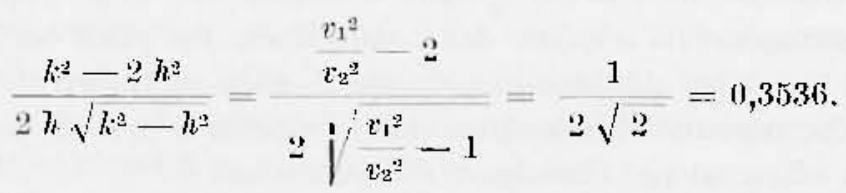

C'on l'ammenture rlella distanza $x$, l'ampiezza di ciasema parte rliminuisce rome $x^{-3 / 2}$, consideranto the in wn solidto illimitato la legge is $x^{1 / 2}$.

1)all'esame delle registrazioni dei numosismi ottenute denturo la

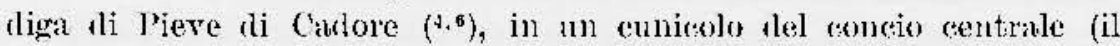
$\mathrm{X}$ [V), risulta che la componente predominunte, in moslo netto, i la valle-monte; trascumbile la destra-sinistra e comunque piccola la verticale. (io dimostm ehe tali mierosismi rientrano nella frungia 

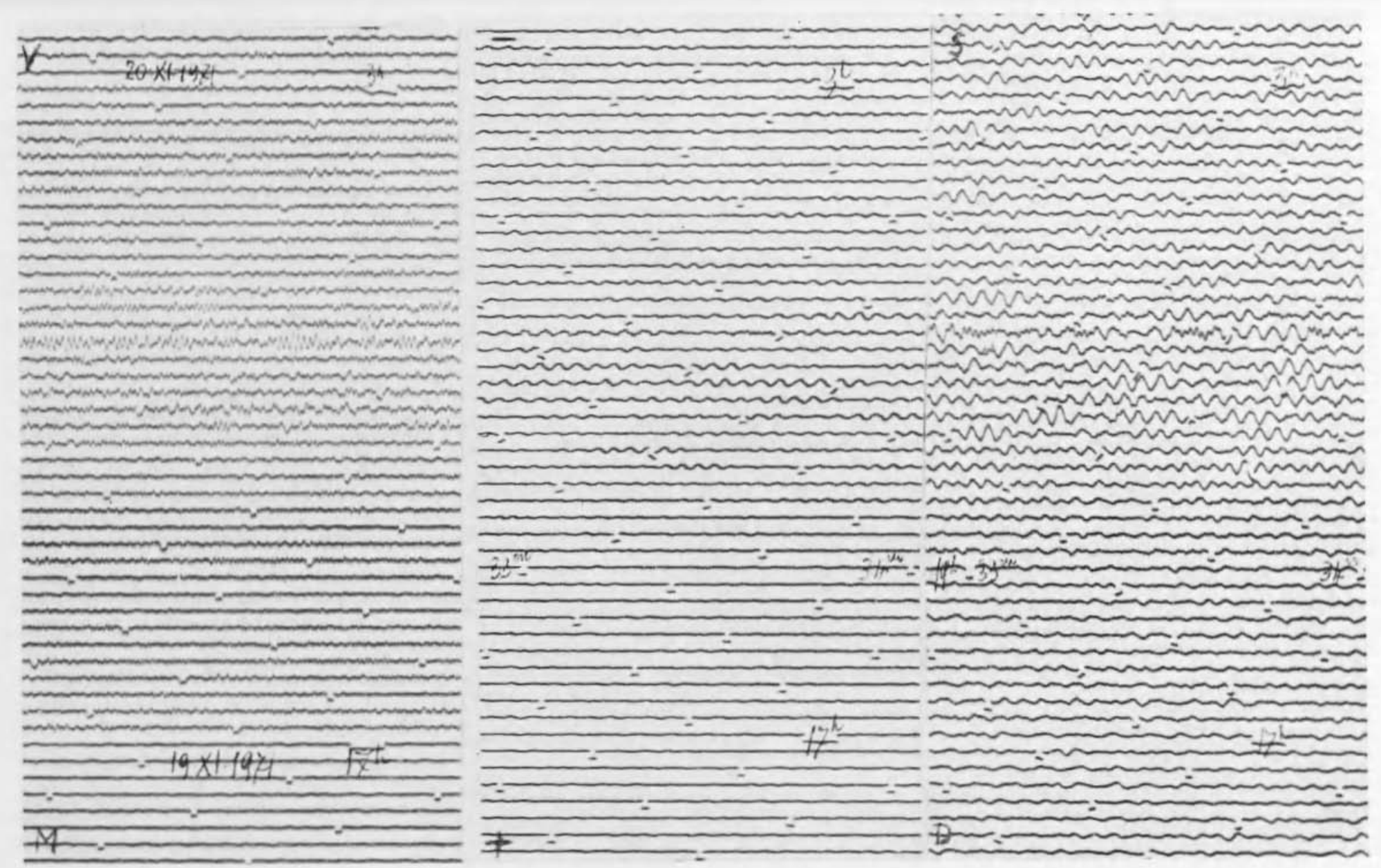

Fig. 1 - Microsismi di carattere locale $(f<1$ s), provoeati da listurbi microharici fransitanti sul lagn di Pieve di ('alore (4.6), soxrapposti a microsismi li origine adriatica $(T=25,8)$, causati da una depressione in transito sul golfo di Trieste. [Ta couj].

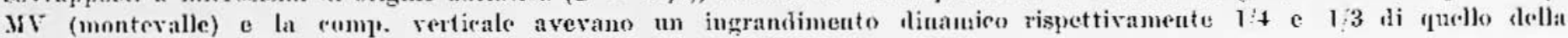
comp. 1)ล (destra-sinistra)]. 
Roma C.U. $\quad 2 \cdot \times 11 \cdot 1972$

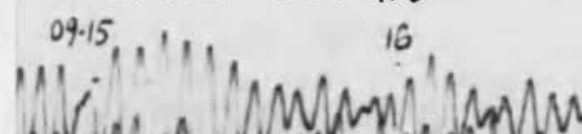

18

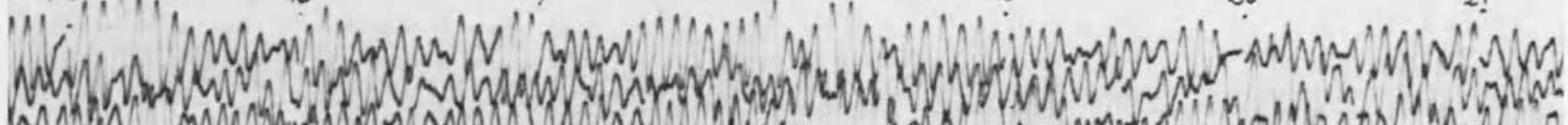

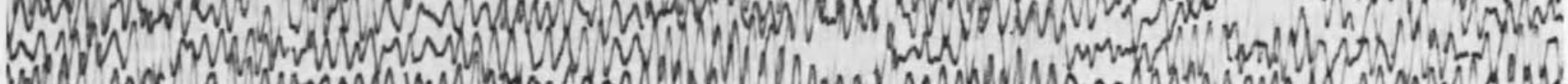

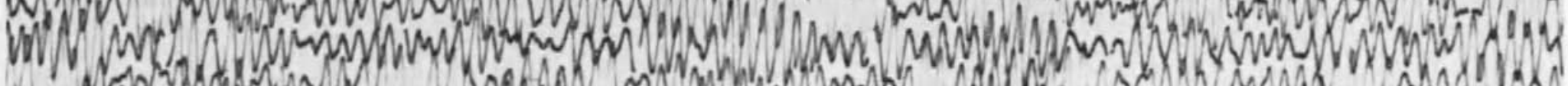
by

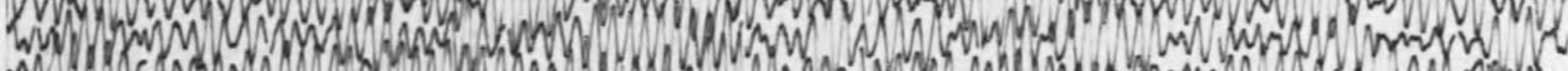

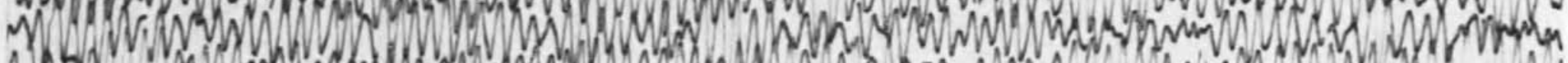

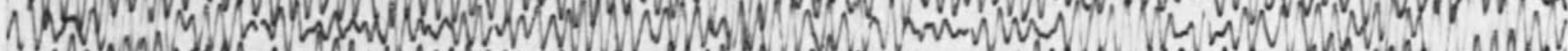

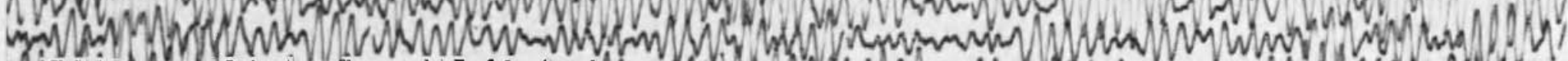
- f

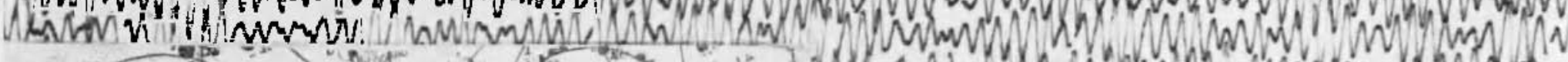

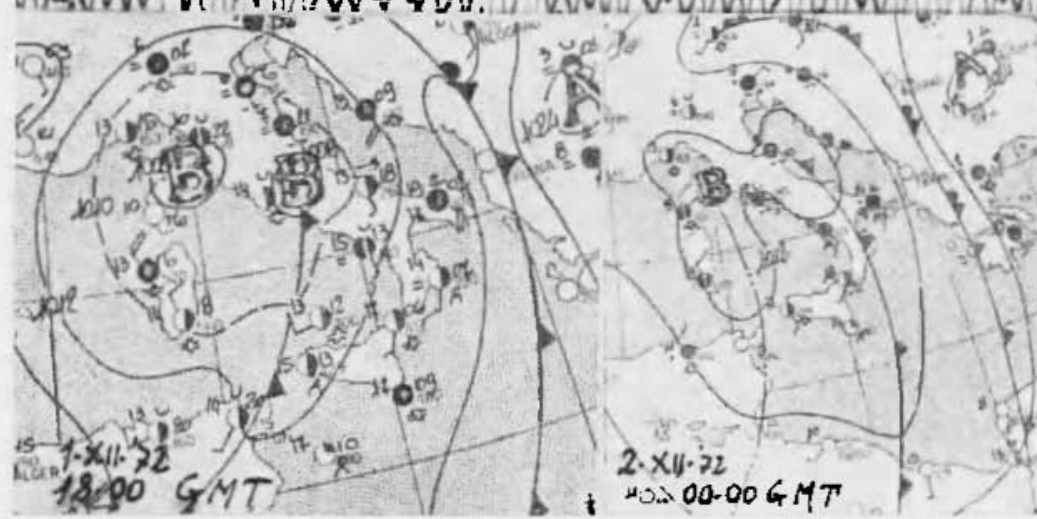

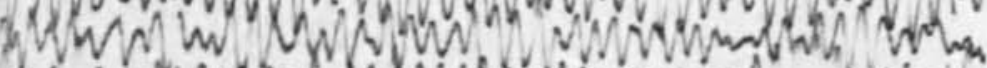

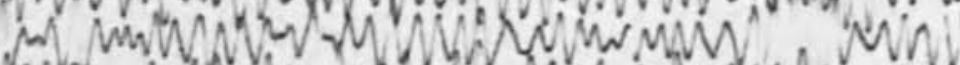

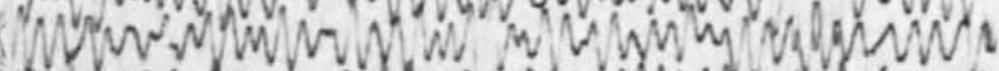

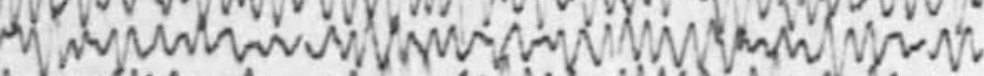

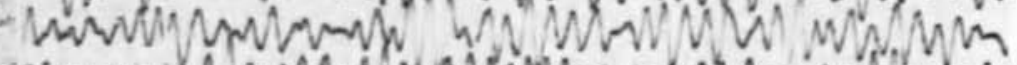

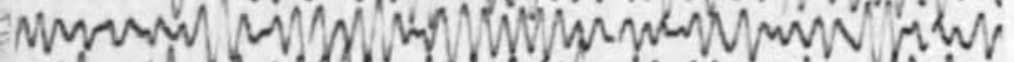

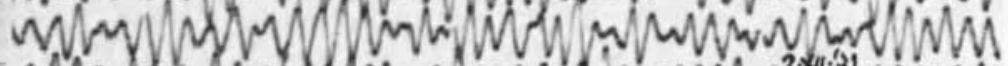

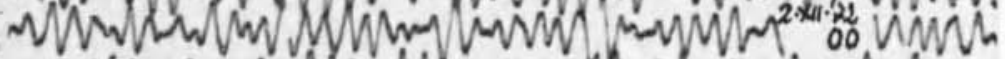

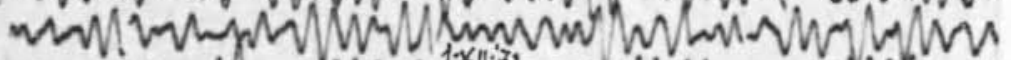

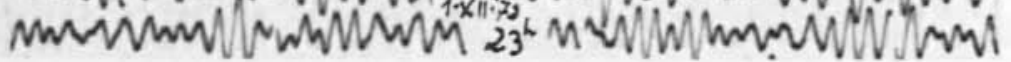

Fig. 2 - Generalmente, i mierosismi provenienti dalla znna di mare intornn all'isola d'Elba hanno perioli tell'nrline di fs, come nel easn in figura. Quando l'area repressionaria è più profondia c più attiva, interessando più estese zone di uare,

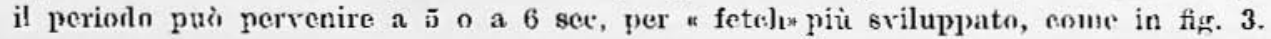




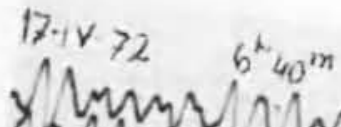

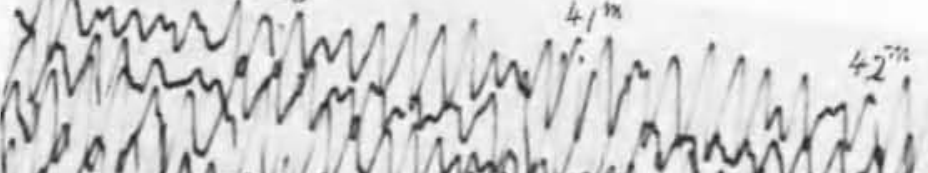

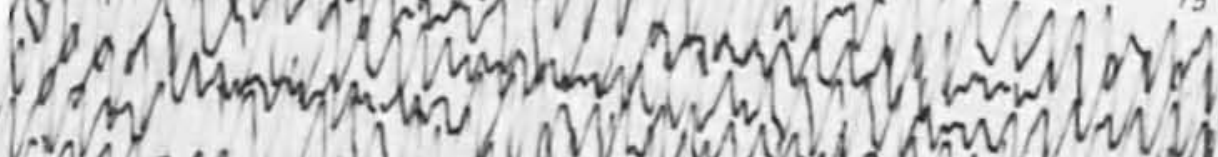

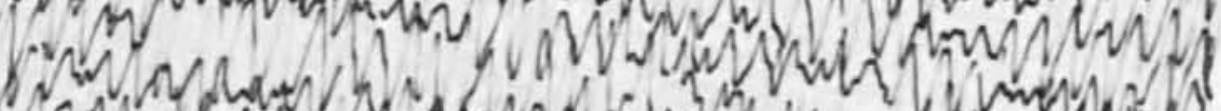

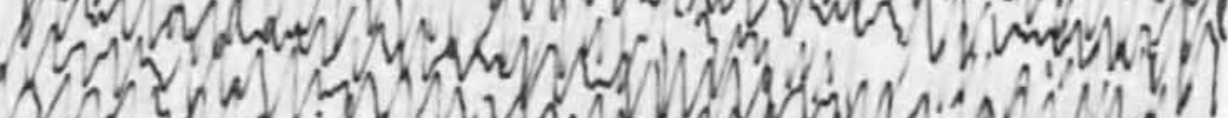

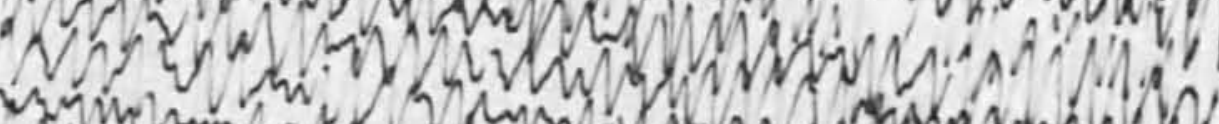

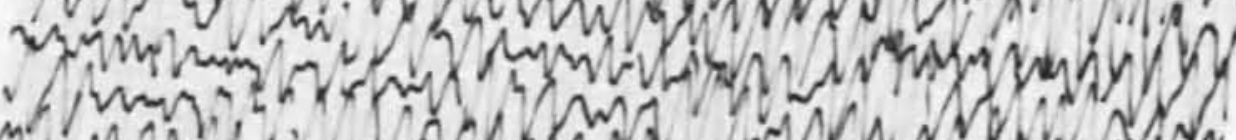

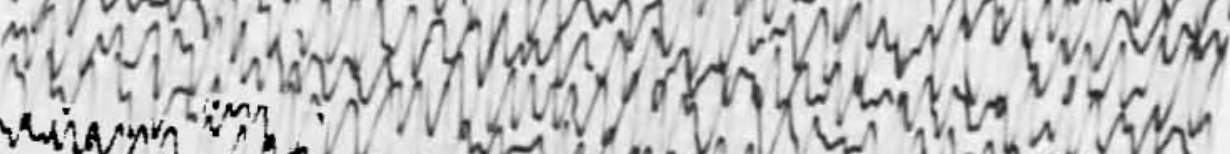

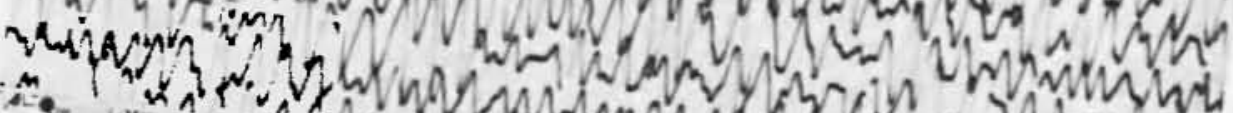

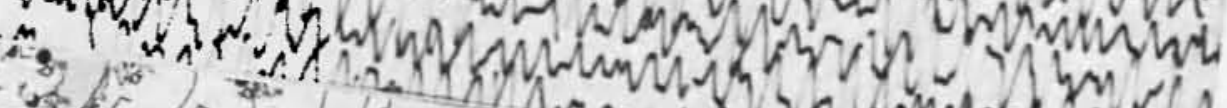
3.0.

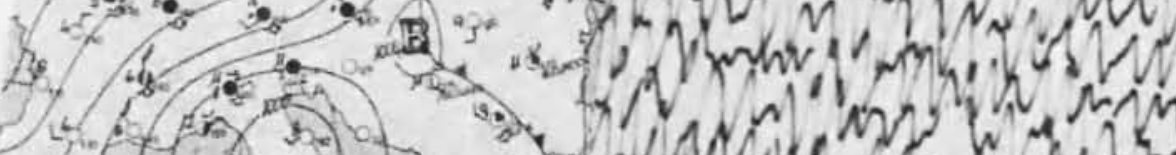

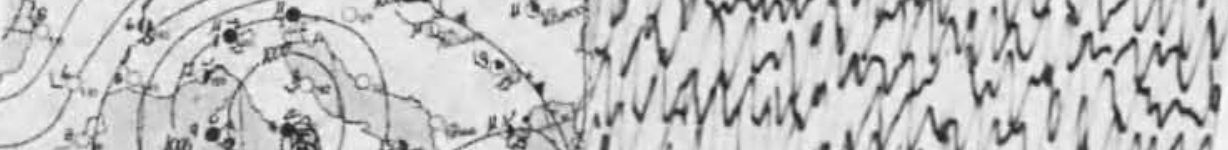

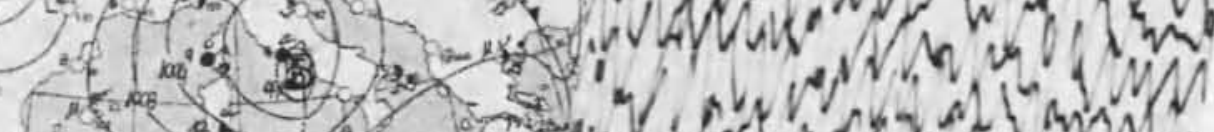

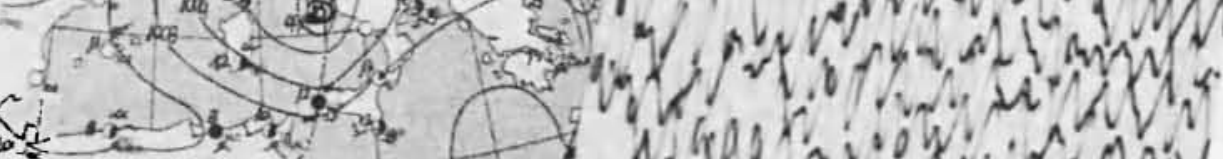

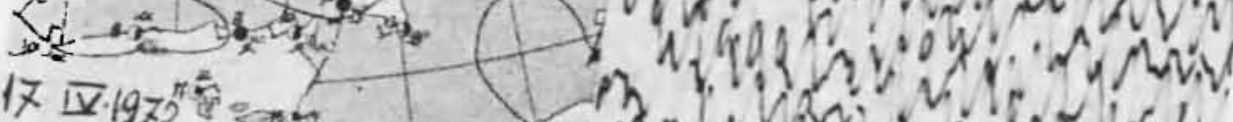

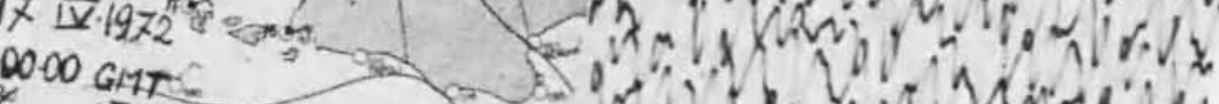
* 72 . whe

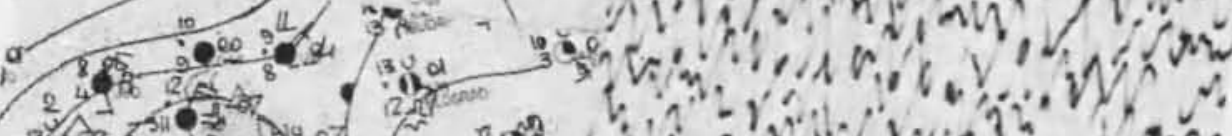
3. 30

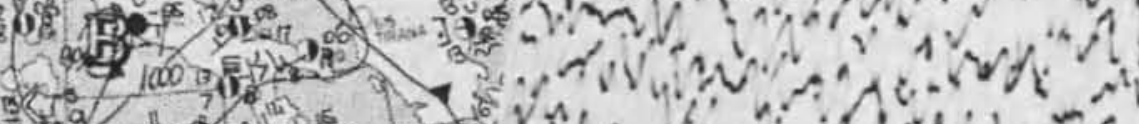

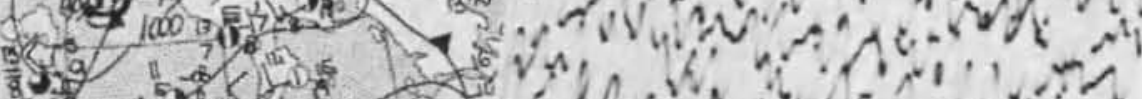

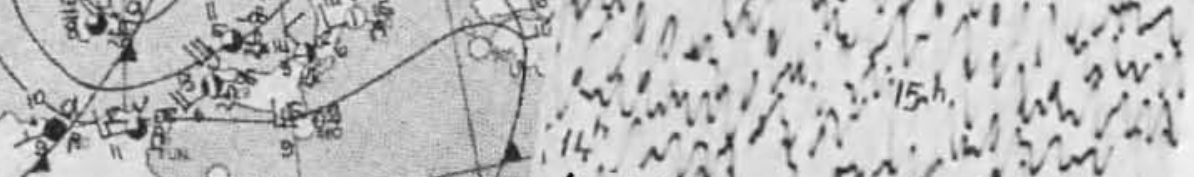
D. 
Roma C.U.

1.iil 19\%2 ws Galitin-Wiht
$7^{m} 36^{\text {mn }}$
$37^{3 n}$
$33^{n}$
$3.9^{\text {th }}$
$40^{n+2}$
$41^{m}$

(2)

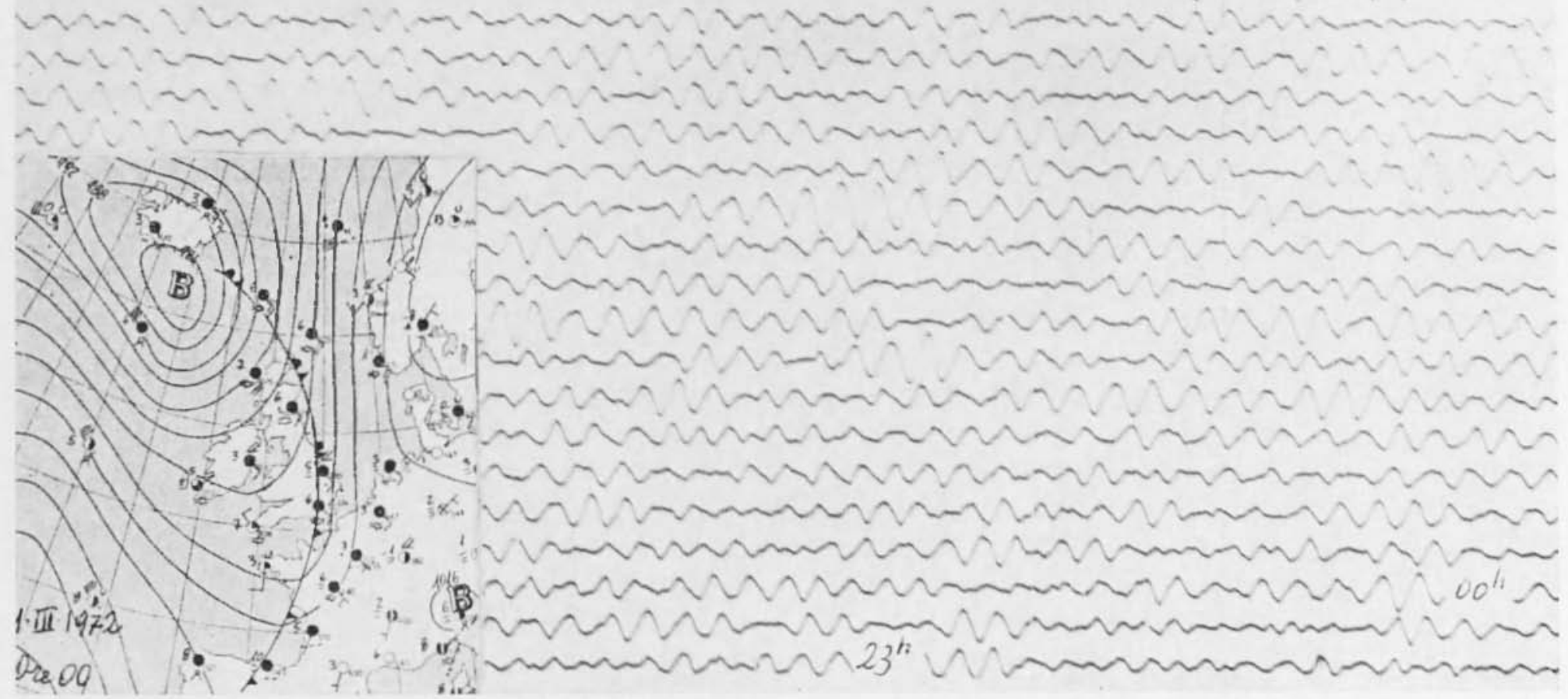

Fig. 4 - I mierosismi provenienti la zone cicloniche in transito sulla fascia atlantica al largo ilelle isole Britanniche, sono generalmente caratterizzati da periodi dell'oriline di 8 see $(5,6)$. Talora, tali perionli possono pervenire a valori dell oriline di 5 sec, coune nell'esempio sopra riportato. 
presente in zoma origrine $t$, in purticolate, ne rostituiscono lat secondat fase, cioc le onde longitudinali. ("iò it provato anche dal valore del rapporto, ripetutamente el'ettuato fra gli impulsi registrati sulle tre componenti - ancles su quelle funzionanti allesterno della diga, in cabina comandi centralizati -, che conlerma il valore sopra riportato, risultanulo compreso flua 0,33 e 0,36 (v. p. es. figgr. 1 , 5). Tel resto, ciò dे

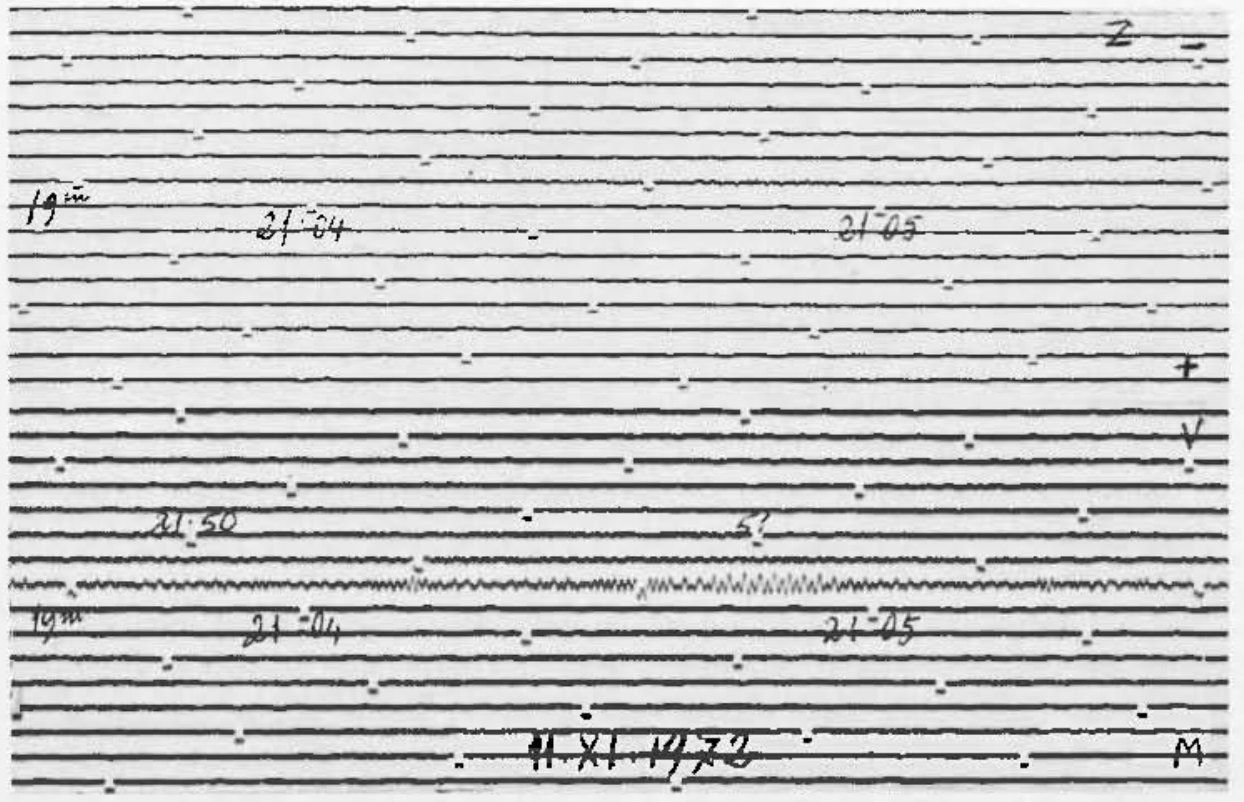

Fir. $\tilde{5}$ - Microsisui di carattere locale, registrati nella digra di l'ieve di Carlore, durante il transito di disturbi microbarici sul lago limitrofus. [Ja com]). VAI (valle-monte) ingranclisce circa i $3: 4$ si ifuella verticale]. Lsoi sono di carattere essenzialmente longitudinale, si spiegano teoricanente come "frange d delle onde di Rayleigl, e interessano la fola zona oririne, conformemente alla teoria di Lamb.

conforme alla teoria. fufatti, prescindendo da $\frac{Q}{\mu}$, per un determinato $x$, il rapporto del roefficiente della parte longitulimale $\left(u_{o}\right)_{1}$ a quello della trasversale $\left(u_{0}\right)_{2}$ della eomponente orizzontale della "frat gia " è ulato da

$$
\frac{\left(u_{0}\right)_{1}}{\left(u_{0}\right)_{2}}=\frac{\left(\frac{h}{h}\right)^{3 / 2}}{\left(1-2 \frac{h^{2}}{k-1}\right)^{3}}
$$




$$
\begin{aligned}
& \text { Per } a=\frac{1}{4} \text { si ha } \\
& \qquad \frac{\left(u_{a}\right)_{1}}{\left(u_{0}\right)_{2}}=\frac{27}{(3 \sqrt{3})^{1 / 2}}=11,84 .
\end{aligned}
$$

Inalogamente, per il rapporto della parte longitudinale $\left(v_{o}\right)_{1}$ della componente verticale, a quella trasversale $\left(v_{o}\right)_{2}$ ciella cosi detta frangia ̀े

$$
\frac{\left(v_{0}\right)_{1}}{\left(v_{0}\right)_{2}}-\frac{1}{1} \frac{\left(\frac{h}{k}\right)^{1 / 2}}{\left(1-2 \frac{h^{2}}{k^{2}}\right)^{2}\left(1-\frac{h^{2}}{k^{2}}\right)} ;
$$

(2, per $\hat{\lambda}=\mu$,

$$
\frac{\left(v_{0}\right)_{1}}{\left(v_{0}\right)_{2}}=\frac{1}{8}\left(\frac{1}{\sqrt{3}}\right)^{1 / 2} \cdot 27=2,564 .
$$

Quindi, la parte longiturlinale della "frangia " ha una componente verticale quasi tripla di quella della parte trasversale; mentre la relativa componente orizzontale è eirea 12 volte quella della parte trasversale.

Concludendo, a piccola distanza dalle zone origini di microsismi, la frangia associata ai movimenti fondamentali (che mancano, come a Pieve di Cadore, dove non sussistono le condizioni per la creazione del "fetch " o dei sistemi di onde significative) è praticamente costituita da sole onde longitudinali. I microsismi sporadici, accidentali, di carattere esclusivamente locale, che insorgono contemporaneamente ai mierosismi fondamentali, destinati alle grandi distanze, sono quindi essenzialmente costituiti ta onde longitudinali.

\section{BIBI.IOGRAFIA}

(1) ANDREotti G., 1932. - Ondulazioni regolari nell'Adriatico e loro azione sul fondo del mare. " $\Lambda$ tti tlel R. Ist. Ven. Scienze etl Arti " Tono XCI, P. II.

(2) Canos P., 1950. - Due earatteristiei tipi di microsismi. "Annali di Geofisica ", III, 3. 
(3) Cano1 P., 1951. - Sullorigine dei microsismi con particolare riguardo all'allo dariatico. "Annali di Geofisican, IV.

(4) CaLoI P., 1971. .. Liagiazione microsismica destata dalle perturbazioni meterologiche: I. Mierosismi It piccoli bacini ehiusi. "Rentl. Aec. Naz. Line oi o. Classe Se. fis., mat. e nat., serie Vlll, L (Magrio).

(5) Calor P., 197]. - Lagitazione microsismiea destata dalle perturbazioni meteorologiche: 11 . Origine dei mierosismi assoriati al trunsito dei ci. clomi. "Rend. Aee. Naz. lincei ". Classe se. fis., mat. e nat., kerie VIII, L (Giugno).

(6) CaLOL 1'., MIGANi ML., 1971. - Mierosismi da piccoli baeini ehinsi, da mari interni, da Oceoni. "Annali di Geofisiea", XXIV, 4.

(7) Deacox G. L. R., 1958 . - Te onde delloceano. "Einteavour ", XVII, 67, 1. 138 .

(8) 1)0w W. I., 1967. - Natural Infrasound of Fine Seconds Period. "Nature: , 215, 5109, pp. 1469-1473.

(9) Kwisg A. W., I'Ress $\mathrm{F}^{4}$, 1952. - Propagation of Elastic ITaves in the Ocen with reference to Microseisms. "Pontificiac Academiae Sciontiarum Seripta Varian. 'Semaine d'Jitude sur le Probleme des Microséismes', p. 124.

(10) LAsus II, 1904, - On the Propagation of Tremors over the Surface of an Elastic Solid. "Philosoplical Transactions", CCIII-A.

(11) latiman G. V., Nownoozi A. A., 1968. - Waves, Wealker, and Onean Bottom. Hicroseisms. "Journal of Geophysical Researchn, 73, 12.

(12) STovbur R., 1952. - The Communication of Energy from Ocen Wanes to the Ocen Floor. "Pontificiae Acalemiae Scientiarum Seripta Va. ria ". "Semaine I'Élue sury le Problène des Microsćismes'. 